\title{
Die Bedeutung des Flexner-Reports für die ärztliche Ausbildung
}

\section{Nathalie Koch}

Dr. med., Psychiatrie und Psychotherapie, MAS Health economics and management

Letztes Jahr fand in der Schweiz die Akkreditierung der medizinischen Aus- und Weiterbildungsgänge statt. Da bietet sich der Rückblick auf die Biographie des US-amerikanischen Bildungswissenschaftlers Abraham Flexner und seinen Bericht über die ärztliche Ausbildung an, den er 1910 im Auftrag der Carnegie-Stiftung (Carnegie Foundation for the Advancement of Teaching) geschrieben hat, den sogenannten «Flexner-Report». Dieses Essay geht den folgenden Fragen nach: Wer war Abraham Flexner? Wie kam er zum Auftrag einer philanthropischen Stiftung, einen Bericht über die ärztliche Ausbildung zu schreiben? Welche Bedeutung hatte der Flexner-Report für die Geschichte der ärztlichen Ausbildung?

\section{Wer war Abraham Flexner?}

Abraham Flexner wurde 1866 in Louisville (Kentucky) als sechstes von neun Kindern aus Deutschland eingewanderter jüdischer Eltern geboren [1]. In der Finanzkrise von 1873 verlor sein Vater sein ganzes Ver-

\section{Zusammenfassung}

Der Bericht, den der US-amerikanische Bildungswissenschaftler Abraham Flexner 1910 über die ärztliche Ausbildung geschrieben hat, der sogenannte "Flexner-Report», hatte eine grosse Bedeutung für die weitere Entwicklung der ärztlichen Ausbildung weltweit. Dieser Artikel fasst die Biographie von Abraham Flexner zusammen und beschreibt, wie er zum Auftrag der Carnegie-Stiftung (Carnegie Foundation for the Advancement of Teaching) gekommen ist, diesen Bericht zu schreiben. Die Wirkung des Flexner-Reports in der Öffentlichkeit war sehr gross. In den Jahren danach wurden in den USA und Kanada viele Ausbildungsstätten für Ärzte geschlossen oder tiefgehend reformiert, das Lernen am Patientenbett erhielt eine Schlüsselrolle, und die US-amerikanischen medizinischen Fakultäten bekamen ihre dreifache Mission von Klinik, Lehre und Forschung. Diese Veränderungen wurden nicht von Flexner alleine angestossen, auch wenn seine Hartnäckigkeit und sein politischer Wille sehr halfen. Sein Bericht hat als Katalysator die Reformen, die schon in den Jahrzehnten davor aufgegleist worden waren, beschleunigt. Er erschien in einer Zeit, in der sich die Öffentlichkeit der grossen Fortschritte der Medizin bewusst wurde und in der die amerikanische Gesellschaft Veränderungen begrüsste. mögen. Alle Kinder mussten so schnell wie möglich arbeiten, um die Haushaltskosten mitzutragen, was sie alle sehr prägte. Sie strengten sich lebenslang an, so erfolgreich wie möglich zu werden.

Sein ältester Bruder, Jacob, ermöglichte es ihm, zwei kurze Jahre an der Johns-Hopkins-Universität zu studieren. Es war die beste amerikanische Universität jener Zeit, die erst acht Jahre davor gegründet worden war. Er belegte so viele Kurse wie möglich und hatte das Gefühl, an einer tiefgreifenden Veränderung des Bildungssystems der USA teilzunehmen. Zurück in Louisville, arbeitete er als Lehrer an einer High School. Als Nebenverdienst bereitete er mit viel Erfolg Schüler im Einzelunterricht auf das College vor [1]. Daraus wurde eine Privatschule, ein Familienunternehmen mit zwei seiner Schwestern als Lehrerinnen. Seine Hoffnung, ein Studium abzuschliessen und Grösseres zu erreichen, gab er jedoch nie auf. Sein Bruder Simon, dem er das Medizinstudium an der Johns-HopkinsUniversität ermöglichte, wurde später zu einem bedeutenden Pathologen. Die Lebenswege dieser beiden Brüder waren eng ineinander verwoben. Abraham las sehr viel, beschäftigte sich mit politischen Belangen und veröffentlichte Artikel in lokalen Zeitungen. Der Präsident der Harvard-Universität, Charles W. Eliot, wurde auf Flexner aufmerksam, da dieser ihm besonders junge und kompetente Studenten empfahl. Eliot ermunterte ihn, seine innovativen Ideen im Bereich 


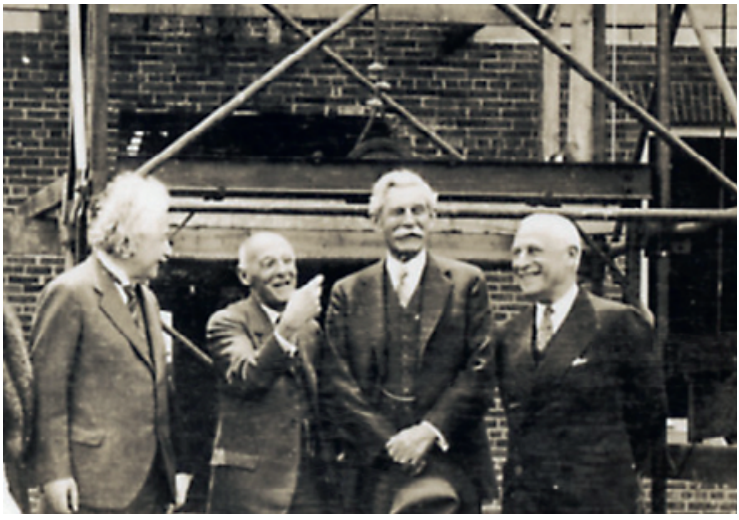

Einstein, Flexner, John R. Hardin und Herbert Maass bei der Grundsteinlegung vom 22. Mai 1939 am Institute for Advanced Study.

der Pädagogik zu veröffentlichen und sollte ihn später als einflussreiches Mitglied des General Education Board der Rockefeller-Stiftung unterstützen. 1898 heiratete Flexner Anne Laziere Crawford, die als Bühnenautorin am Broadway finanziell abgesichert war. 1905 konnte er seine Privatschule verkaufen und bekam endlich die Gelegenheit, sein Studium weiterzuführen [1]. Er studierte ein Jahr in Harvard sowie zwei Jahre in Berlin und Heidelberg. Zurück in Amerika, verbrachte er den Rest seines Lebens in New York, wo sein Bruder Simon unterdessen Direktor des Rockefeller-Instituts für Medizinische Forschung geworden war. 1908 verfasste Abraham sein erstes Buch, The American College: a Criticism, in dem er die amerikanischen Colleges heftig kritisierte. Die Carnegie-Stiftung gab ihm daraufhin den Auftrag, einen Bericht zur ärztlichen Ausbildung zu verfassen. Später arbeitete er 15 Jahre für die Rockefeller-Stiftung und wirkte ab 1930 als Gründungsdirektor des Institute for Advanced Study in Princeton, an das er den 1933 aus Nazi-Deutschland emigrierten Albert Einstein sowie andere geflohene Wissenschaftler holte. Flexner starb 1959.

\section{Die medizinische Ausbildung Anfang des 20. Jahrhunderts}

In der zweiten Hälfte des 19. Jahrhunderts machte die Medizin wichtige Fortschritte. Die ärztliche Tätigkeit veränderte sich nachhaltig durch die Entdeckung von Bakterien als Erreger übertragbarer Krankheiten, neue Mittel zur Vermeidung von Infektionen sowie die Einführung von verschiedenen Instrumenten und Labortechniken zur Diagnose von Krankheiten [2]. Die Medizin wurde zu einer klinischen Wissenschaft. Das dynamische Wissen von wissenschaftlichen Zeitschriften ersetzte zunehmend das statische Wissen in Büchern. Medizinische Fachgesellschaften traten immer selbstbewusster in der Berufs- und Wissenschaftspolitik auf [3]. Spitäler waren nun immer weniger Asyle für Arme und Unheilbare und immer mehr Einrichtungen zur Therapie heilbarer Krankheiten. Sie etablierten sich auch zunehmend als Zentren der ärztlichen Ausbildung. Die neuen Erkenntnisse mussten in die Ausbildung aufgenommen werden, die immer umfassender wurde.

In den Vereinigten Staaten existierten medizinische Ausbildungsgänge seit dem 18. Jahrhundert. Bis Anfang des 20. Jahrhunderts waren sie wenig vereinheitlicht. Private Schulen, typischerweise von fünf bis sieben Ärzten geführt, ohne Bezug zu Universitäten und Spitälern, verkauften Kurse an Studenten mit oft mangelhafter Vorbildung, vorausgesetzt, dass sie bezahlen konnten [4]. Die Dürftigkeit der Ausbildung der Ärzte wurde ab Mitte des 19. Jahrhunderts immer offensichtlicher, sie genügte den in Europa etablierten wissenschaftlichen und pädagogischen Standards nicht mehr. 1876 etablierte die Johns-Hopkins-Universität die erste medizinische Fakultät nach deutschem Vorbild und integrierte das Spital in die medizinische Fakultät. Sie spielte danach eine führende Rolle in der Reform der medizinischen Ausbildung und erarbeitete zusammen mit der American Medical Association (AMA) in einer Pilotstudie die wesentlichen Punkte einer Reform, die von den Colleges im ganzen Land umgesetzt werden sollte.

\section{Der Flexner-Report}

In Absprache mit dem Council of Medical Education der AMA beschloss 1908 die Carnegie-Stiftung, eine detaillierte Studie über die nordamerikanische ärztliche Ausbildung zu verfassen. Deren Präsident, Henry S. Pritchett, bot Abraham Flexner an, den Bericht zu schreiben, damit er aus der Sicht eines unvoreingenommenen und kritischen Pädagogen und nicht von Ärzten verfasst würde [2]. Die AMA verheimlichte ihre Zusammenarbeit, da die Carnegie-Stiftung eine neutrale Instanz darstellen sollte. Ab 1908 besuchte Flexner innerhalb von acht Monaten 155 medizinische Ausbildungsstätten in den USA und Kanada, einige von ihnen mehrmals. Er überprüfte sie auf Aufnahmekriterien, die Anzahl der Professoren im Verhältnis zur Studentenschaft, Finanzen, das Vorhandensein von Laboratorien und den Zugang zu Spitälern [3]. Einen standardisierten Fragebogen zu benützen, war damals noch nicht üblich. Die Schulen öffneten ihm ihre Türen, da sie hofften, danach Geld für Reformen von der Carnegie-Stiftung zu bekommen [1]. Einige baten während der Besuche um Ratschläge, wie sie sich verbessern konnten. 


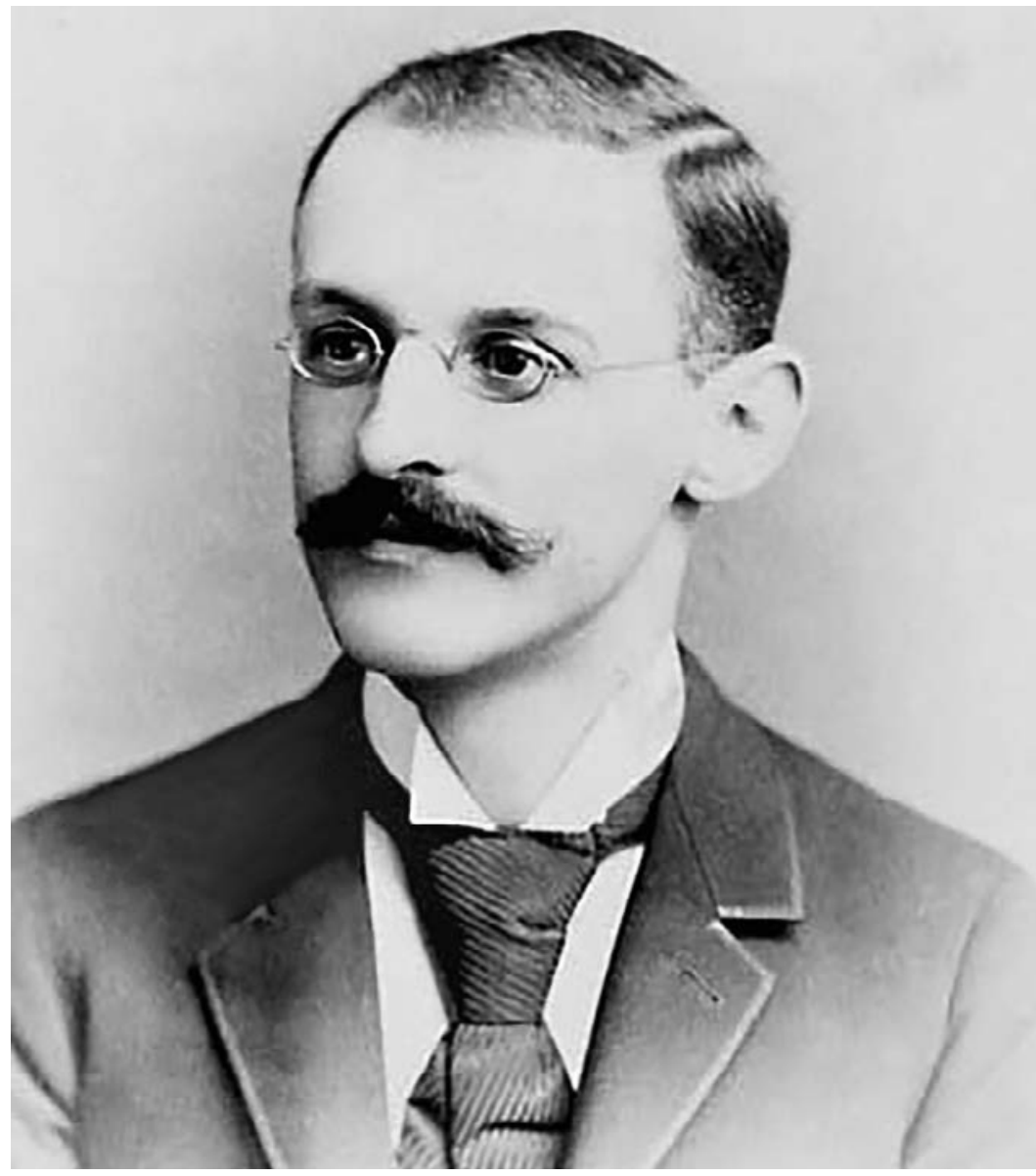

Abraham Flexner (1866-1959) im Jahr 1895.

Bei der Redaktion des Berichts des Flexner-Reports halfen bedeutende Ärzte mit, insbesondere sein Bruder Simon und William Welch, der erste Dekan der medizinischen Fakultät der Johns-Hopkins-Universität. Der Bericht empfahl, 117 von 148 Medical Schools in Nordamerika im Interesse des öffentlichen Wohls zu schliessen. Grundsätzlich sollten weniger, aber qualitativ bessere Ärzte ausgebildet werden. Die Ausbildung sollte in die Universitäten eingegliedert werden und sich am wissenschaftlichen Stand orientieren. Die Ausbildungsstätten sollten über Laboratorien und Spitalabteilungen verfügen, um den Zugang sowohl zu den wissenschaftlichen Grundlagen als auch den Patienten zu sichern. Die Ausbildung sollte nicht mehr profitorientiert finanziert werden. Flexner schlug eine neue Struktur der Ausbildungsgänge vor: Die angehenden Ärzte mussten vor der Aufnahme die grundlegenden Naturwissenschaften erlernt haben; das Curriculum musste die grundlegenden Labortechniken (Anatomie, Histologie, Physiologie usw.) in den ersten Jahren beinhalten; in zwei weiteren Jahren musste die aktive klinische Betätigung in Spitälern unter Supervision gewährleistet sein. So wurde die wissenschaftliche Ausbildung mit dem bedside teaching verbunden, und das learning by doing, welches für Flexner höchste Priorität hatte, erhielt eine Schlüsselrolle. Ein Punkt, der bei der Umsetzung die grössten Konflikte mit den Ärzten verursachen würde (unter anderem auch mit dem prominenten William Osler), war für Flexner besonders wichtig: Die Lehrtätigen sollten in Vollzeit unterrichten und forschen und kein Geld mit der Behandlung von Patienten verdienen.

Der Bericht erfuhr nach seiner Veröffentlichung eine sensationelle Aufmerksamkeit in den Medien und der Öffentlichkeit und elektrisierte viele Menschen. Gewisse Colleges machten sich umgehend daran, seine Vorschläge umzusetzen. Andere Colleges beendeten ihren Betrieb. 1920 gab es nur noch halb so viele ärztliche Ausbildungsstätten wie 1900 [2].

\section{Der Einfluss des Flexner-Reports}

Inwiefern die Schliessung vieler Schulen und die Reformen des Medizinstudiums jener Jahre auf den Flexner-Report zurückzuführen sind, ist eine Frage, die lange kontrovers beurteilt wurde. Abraham Flexner verfolgte seine Ziele im Bereich der Bildung mit grossem unternehmerischem Geist. Er war eine umstrittene Persönlichkeit und wurde von vielen Menschen als dogmatisch und autoritär empfunden. Sein Bericht stützte sich auf Reformvorschläge, die schon in Berichten der ärztlichen Colleges sowie der AMA vorher eine wesentliche Rolle eingenommen hatten und die einige führende Universitäten schon umgesetzt hatten [2]. Die grossen Veränderungen wurden durch das Zusammentreffen verschiedener Akteure erreicht: die institutionelle Unterstützung durch die AMA, prominente Professoren, staatliche Regulierungsinstanzen, die Carnegie- und die Rockefeller-Stiftung sowie die Hartnäckigkeit und der politische Wille von Abraham Flexner [5].

Heute besteht ein Konsens, dass der Flexner-Report als Katalysator die Reformen, die schon aufgegleist waren, beschleunigt hat. Er erschien zur richtigen Zeit am richtigen Ort und widerspiegelte die Ansichten vieler Ärzte, die die Ausbildung an die neuesten wissenschaftlichen Erkenntnisse anpassen wollten. Flexner war ein Agent mächtiger Bewegungen, die schon seit einem halben Jahrhundert begonnen hatten. Mehrere Faktoren haben dazu beigetragen: Es gab zu der Zeit relativ wenige Interessengruppen jenseits der AMA; so konnten die Vorschläge der kleinen Gruppe von Reformatoren relativ einfach umgesetzt werden [2]. Da Flexner kein Arzt war, konnte er die qualitativen Mängel der Ausbildung besser ansprechen als die Ärzte selbst. 
Sein direkter Stil mit scharf formulierter Kritik wühlte die Öffentlichkeit auf, er beschrieb Missstände hemmungslos. Er war aber nicht nur das Sprachrohr der Ärzte, die eine Reform wünschten, sondern auch ein überzeugter Pädagoge, der neue Standards für das Wohl der Studierenden und den Schutz der Bevölkerung kreieren wollte. Sein Bericht erschien mitten in der progressiven Ära der amerikanischen Politik, die unter anderem das Bildungssystem erneuern wollte. Es war eine Zeit, in der der Öffentlichkeit bewusst wurde, wie grosse Fortschritte die Medizin machte und welches Potential dahintersteckte [5].

Andrew Carnegie wollte nach Erscheinen des Berichtes nicht mehr Geld in die ärztliche Ausbildung investieren, da er seiner Ansicht nach bestätigte, dass die medizinische Ausbildung vor allem ein Geschäft war [1]. Dafür sah die Rockefeller-Stiftung ein geeignetes Feld für Investitionen und stellte Abraham Flexner ab 1918 als Sekretär seines General Education Board an. Wo im 19. Jahrhundert die meisten ärztlichen Ausbildungsstätten von Studentenbeiträgen lebten und die staatliche Unterstützung bescheiden war, eröffnete sich neu die Möglichkeit, Stiftungsgelder anzuziehen. Flexner verstand es, den Zeitpunkt zu nutzen, um dank seiner Überzeugungskraft Spendengelder in die Reform der ärztlichen Ausbildung fliessen zu lassen [6]. In den nächsten 15 Jahren gelang es ihm, eine Summe von über 500 Millionen Rockefeller-Dollar (sowie viele Gelder anderer wohlhabender Spender) in die Modernisierung der neuen Medical Schools zu steuern.

Der Historiker der ärztlichen Ausbildung Kenneth M. Ludmerer, aus dessen Sicht der Einfluss von Flexner allgemein überschätzt wird, bezeichnet die Reformwelle als Revolution, in der ein neuer gesellschaftlicher Vertrag zustande kam: Die Gesellschaft musste die finanzielle, politische und moralische Unterstützung sicherstellen für die ärztliche Bildung und Forschung. Im Gegenzug mussten die Medical Schools der Öffentlichkeit dienen, indem sie die Qualität der Forschung und der ärztlichen Versorgung sicherstellten [7]. So bekamen die amerikanischen medizinischen Fakultäten die dreifache Mission, die an Universitätsspitälern weltweit heute noch gilt: Klinik, Lehre und Forschung.

\section{Schlusswort}

Vor über hundert Jahren wurde die medizinische Ausbildung durch ein Zusammentreffen wissenschaft- licher, sozialer, politischer und ökonomischer Umstände tiefgreifend verändert. Seither wird ihr eher vorgeworfen, sich zu sehr am (natur-)wissenschaftlichen Stand $\mathrm{zu}$ orientieren und das ganzheitliche Menschenbild sowie die öffentliche Gesundheit zu vernachlässigen [8]. Die Frage der Verfügbarkeit der Professoren und Supervisoren stellt sich immer wieder erneut. Der Druck auf die Produktivität der Spitäler, die wachsende Komplexität der Behandlungen, die Verkürzung der Spitalaufenthalte und die Beschäftigung mit der Forschung, die sich seither vom Patientenbett entfernt und auf die Grundlagenforschung verlegt hat, können alle die Zeit, die für die ärztliche Aus- und Weiterbildung zur Verfügung steht, beeinträchtigen [8]. Die Interessengruppen haben sich vermehrt, und ihre Beziehungen zueinander sind komplexer geworden. Wenn Abraham Flexner heute noch leben würde, würde er vermutlich die laufenden Veränderungen tatkräftig unterstützen [8].

\section{Bildnachweise}

Gruppenfoto: Shelby White and Leon Levy Archives Center, Institute for Advanced Study, Princeton, NJ, USA, https://commons.wikimedia. org/wiki/File:Albert_Einstein_and_Abraham_Flexner_at_the_Institute for Advanced Study.jpg

Porträt Flexner: https://de.wikipedia.org/wiki/Abraham_Flexner\#/ media/File:Aflexner21.jpg, gemeinfrei

\section{Disclosure statement}

Keine Interessenverbindungen. Arbeit im Rahmen des CAS Applied History der Universität Zürich.

\section{Literatur}

1 Bonner TN. Iconoclast: Abraham Flexner and a Life in Learning. 2002: Johns Hopkins University Press. eBook edition, Plunkett Lake Press; 2016.

2 Barzansky B. Abraham Flexner and the era of medical education reform. Acad Med. 2010;85(9 Suppl):19-25.

3 Calman K. Medical Education: Past, Present and Future - Handing on learning, E. Churchill Livingstone; 2006.

4 Miller LE, Weiss RM. Medical education reform efforts and failures of U.S. medical schools, 1870-1930. J Hist Med Allied Sci. 2008; 63(3):348-87.

5 Halperin EC, Perman JA, Wilson EA. Abraham Flexner of Kentucky, his report, Medical Education in the United States and Canada, and the historical questions raised by the report. Acad Med. 2010; 85(2):203-10.

6 Markel H. Abraham Flexner and his remarkable report on medical education: a century later. JAMA. 2010;303(9):888-90.

7 Ludmerer KM. Time to Heal-American medical education from the turn of the century to the era of managed care. Oxford University Press; 1999.

8 Cooke M, Irby DM, Sullivan W, Ludmerer KM. American medical education 100 years after the Flexner report. N Engl J Med. 2006; 355(13):1339-44. 